\title{
Patient and health system costs of managing pregnancy and birth-related complications in sub-Saharan Africa: a systematic review
}

Amani Thomas Mori ${ }^{1,2,3^{*}}$ (D), Peter Binyaruka ${ }^{4}$, Peter Hangoma ${ }^{5}$, Bjarne Robberstad ${ }^{1,2,3}$ and Ingvild Sandoy ${ }^{1,3}$

\begin{abstract}
Background: Morbidity and mortality due to pregnancy and childbearing are high in developing countries. This study aims to estimate patient and health system costs of managing pregnancy and birth-related complications in sub-Saharan Africa.

Methods: A systematic review of the literature was conducted to identify costing studies published and unpublished, from January 2000 to May 2019. The search was done in Pubmed, EMBASE, Cinahl, and Web of Science databases and grey literature. The study was registered in PROSPERO with registration No.

CRD42019119316. All costs were converted to 2018 US dollars using relevant Consumer Price Indices.

Results: Out of 1652 studies identified, 48 fulfilled the inclusion criteria. The included studies were of moderate to high quality. Spontaneous vaginal delivery cost patients and health systems between USD 6-52 and USD 8-73, but cesarean section costs between USD 56-377 and USD 80-562, respectively. Patient and health system costs of abortion range between USD 11-66 and USD 40-298, while post-abortion care costs between USD 21-158 and USD 46-151, respectively. The patient and health system costs for managing a case of eclampsia range between USD 52-231 and USD 123-186, while for maternal hemorrhage they range between USD 65-196 and USD 30-127, respectively. Patient cost for caring low-birth weight babies ranges between USD 38-489 while the health system cost was estimated to be USD 514.

Conclusion: This is the first systematic review to compile comprehensive up-to-date patient and health system costs of managing pregnancy and birth-related complications in sub-Saharan Africa. It indicates that these costs are relatively high in this region and that patient costs were largely catastrophic relative to a $10 \%$ of average national per capita income.
\end{abstract}

Keywords: Pregnancy, Birth, Maternal complications, Cost, Catastrophic health expenditure

\footnotetext{
* Correspondence: pax_amani@yahoo.com

${ }^{1}$ Centre for International Health, University of Bergen, P.O. Box 7804, 5020 Bergen, Norway

${ }^{2}$ Department of Global Public Health and Primary Care, Section for Ethics and Health Economics, University of Bergen, Bergen, Norway

Full list of author information is available at the end of the article
}

(c) The Author(s). 2020 Open Access This article is licensed under a Creative Commons Attribution 4.0 International License, which permits use, sharing, adaptation, distribution and reproduction in any medium or format, as long as you give appropriate credit to the original author(s) and the source, provide a link to the Creative Commons licence, and indicate if changes were made. The images or other third party material in this article are included in the article's Creative Commons. licence, unless indicated otherwise in a credit line to the material. If material is not included in the article's Creative Commons licence and your intended use is not permitted by statutory regulation or exceeds the permitted use, you will need to obtain permission directly from the copyright holder. To view a copy of this licence, visit http://creativecommons.org/licenses/by/4.0/ The Creative Commons Public Domain Dedication waiver (http://creativecommons.org/publicdomain/zero/1.0/) applies to the data made available in this article, unless otherwise stated in a credit line to the data. 


\section{Introduction}

An estimated 303,000 preventable deaths occurred during pregnancy and childbirth globally in 2015 , mostly as a result of pregnancy and birth-related complications. Most of these maternal deaths occurred in low-income countries, particularly in sub-Saharan Africa. About three-quarters of these complications include unsafe abortions, hypertensive disorders in pregnancy i.e. pre-eclampsia and eclampsia, sepsis, severe bleeding, and complications arising at the time of delivery [1, 2]. Globally, about 17 million girls aged less than 19 years give births every year, and about 4 million undergo unsafe abortions to terminate unwanted pregnancies, and these adolescent pregnancies are associated with elevated risks of complications [1-3]. Pregnancy and childbearing complications are ranked fourth globally and second in low- and middle-income countries among the leading causes of death in adolescent girls [4].

The United Nations Development Fund reports that the prevalence of adolescent pregnancy has decreased globally, but remained relatively unchanged in subSaharan Africa [5]. By 2030, it is expected that the population of adolescent girls in sub-Saharan Africa will grow by $50 \%$; hence, escalating the problem of teen pregnancy and childbearing [5]. Since adolescent pregnancy is associated with elevated risk of complications [1-3], it is likely that the total costs of treating pregnancy and childbearing complications in sub-Saharan Africa will also increase. The cost of pregnancy and birth-related complications have been synthesized and documented in systematic reviews conducted elsewhere [6-8] but not in sub-Saharan Africa despite being the region that carries the largest burden of maternal death globally [5].

This study aims to assess patient and health system costs associated with the management of pregnancy and birth-related complications in sub-Saharan Africa. The results will feed into a cost-benefit analysis study comparing two adolescent pregnancy prevention strategies in Zambia to help policymakers to choose the strategy with the greatest potential for return on investment [9]. The study findings may also be useful to researchers and policymakers elsewhere as it aims to provide cost evidence that can facilitate economic evaluation and budget impact analyses of maternal and child health interventions to demonstrate whether they represent value for money or not in addition to positive public health impact.

\section{Methods}

We used the PRISMA checklist that is recommended for reporting a systematic review and meta-analysis of clinical trials [10], with slight modifications to suit the review of costing studies. The study protocol was registered with PROSPERO-the International Prospective Register of Systematic Reviews with registration No. CRD42019119316.

\section{Search strategy and inclusion criteria}

The search of the literature was conducted by ATM in Pubmed, EMBASE, Cinahl, and Web of Science databases using combinations of the following search terms: cost, costs, cost of illness, economic burden, cost analysis, healthcare costs, health care costs, preterm birth, low birth weight, preeclampsia, eclampsia, abortion, post-abortion complication, cesarean section, and individual names of sub-Saharan African countries. An example of a search code used to search in Pubmed is shown in Table 1. The last search of these databases was conducted on 26th November 2018. However, we allowed Pubmed and Web of Science to send us weekly updates on the saved search terms until 13th May 2019, during which two more qualifying articles were found. Other articles were identified by scanning reference lists of review papers and relevant costing studies and searching with the Google search engine using the abovementioned search terms. We also contacted some authors to ask for unpublished articles.

We included costing studies that i) were conducted in sub-Saharan Africa ii) published from January 2000 to 13th May 2019 iii) targeted normal delivery as well as pregnancy and birth-related complications including

Table 1 Search in PubMed

\begin{tabular}{|c|c|}
\hline No. & Search query \\
\hline$\# 1$ & $\begin{array}{l}\text { (cost) OR "economic cost") OR "economic analysis") OR "economic burden") OR "healthcare cost") OR "cost of illness") OR "health care cost") } \\
\text { OR "patient cost")) }\end{array}$ \\
\hline \#2 & $\begin{array}{l}\text { (eclampsia) OR preeclampsia) OR pre-eclampsia) OR "pre eclampsia") OR "preterm birth") OR "pre-term birth") OR premature) OR "low birth } \\
\text { weight") OR low-birth weight) OR "lowbirth weight") OR "small for gestational age") OR still-birth) OR stillbirth) OR abortion) OR "post } \\
\text { abortion complication") OR c-section) OR "cesarean section")) }\end{array}$ \\
\hline \#3 & $\begin{array}{l}\text { (Angola) OR Benin) OR Botswana) OR Burkina Faso) OR Burundi) OR (ameroon) OR (ape Verde) OR (entral African Republic) OR (had) OR } \\
\text { Comoros) OR Congo) OR Cote d'Ivoire) OR Djibouti) OR Equatorial Guinea) OR Eritrea) OR Gabon) OR Ethiopia) OR The Gambia) OR Ghana) } \\
\text { OR Guinea) OR Guinea-Bissau) OR Kenya) OR Lesotho) OR Liberia) OR Malawi) OR Madagascar) OR Mali) OR Mauritania) OR Mauritius) OR } \\
\text { Mozambique) OR Namibia) OR Niger) OR Nigeria) OR Rwanda) OR Reunion) OR (Sao Tome and Principles)) OR Senegal) OR Seychelles) OR } \\
\text { Sudan) OR Sierra Leone) OR Somalia) OR South Africa) OR Swaziland) OR Tanzania) OR Togo) OR Uganda) OR Western Sahara) OR Zambia) } \\
\text { OR Zimbabwe) }\end{array}$ \\
\hline \#4 & \#1 AND \#2 AND \#3 \\
\hline
\end{tabular}


pre-eclampsia, eclampsia, pre-term birth, low birth weight babies, small for gestational age babies, unsafe abortion and post-abortion complications. The search was limited to humans and the English language. Review papers and reports were excluded because we were only interested in primary cost data, but they were instead used to identify other relevant studies. Two reviewers (ATM and PB) independently screened the titles and abstracts of all the articles to assess eligibility and the qualifying ones were subjected to further screening for eligibility by the two reviewers by reading the full text.

\section{Quality assessment}

Quality assessment was conducted independently by ATM and PH. To the best of our knowledge, there is no quality assessment guideline for cost studies: hence, we developed an 8-item checklist from Drummond et al. [11], Liers et al. [12], and the Consolidated Health Economic Evaluation Reporting Standards (CHEERS) [13]. The 8 items were: i) description of the characteristics of the study population and the reasons why it was chosen; ii) the costing methodology used must be clearly reported, whether micro-costing or gross costing approach or a combination; iii) the sources used to collect resource utilization data should be reported clearly (e.g. clinical trials, administrative databases, clinical databases, medical records and published literature); iv) resource quantities should be reported or described independently from the costs, so that assessment of the measurement method is facilitated; v) the viewpoint/ perspective of the analysis such as the provider, patient and family or societal perspectives should be clearly described;vi) all costs should be adjusted to a specific price year so that the effects of inflation are removed from the cost estimation; vii) If the time horizon for estimating costs was longer than 1 year, discounting should have been performed to reflect time preferences viii) if prices were used instead of costs, they should reflect the true opportunity costs. Quality was assessed by scoring each of the items with a value of 1 if fully completed, 0.5 if not fully completed, 0 if not completed, and NA if not applicable. The quality scores were categorized as 'low' if $\leq 33 \%$, 'moderate' if the score was between 33 and 66\%, and 'high' if $>66 \%$. Disagreements on eligibility or the quality assessments were resolved through consensus.

\section{Data analysis}

We extracted information about the name of the primary author, year of publication, year in which the data was collected, study design, the country in which data was collected, costing perspective used, the currency used, cost information, disease condition, target population from which data was collected and the level of the healthcare facility.
Costs were categorized as health system costs if they were borne by the healthcare facility and patient costs if they were borne by the patient or caregiver. Health system costs could further be categorized as recurrent if spent on items that are used up in the course of the year such as salaries, supplies, and utilities or capital costs if spent on items that last more than a year such as buildings, furniture, and equipment. Patient costs included both direct costs and indirect costs. Direct costs were those paid in the process of seeking/accessing care and included out-of-pocket payment for treatment (registration, diagnosis, radiology, drugs, bed days, etc), transport to and from the healthcare facility, food, and other related expenses. Indirect costs were those that resulted from the loss of income as a result of not being able to engage in economically productive activities due to illness. When costs were reported separately for public, private, or non-governmental organizations, etc., we computed a simple average.

Base year costs in local currencies were first converted to US dollars (USD) using the existing exchange rate for the base years of the individual studies, before adjustment to 2018 USD using relevant US Consumer Price Indices (CPI) [14]. Annual Gross National Income (GNI) per capita was used as a proxy of household income and out of pocket patient payments that exceeded $10 \%$ of this income were assumed to constitute catastrophic health expenditure [15].

\section{Results}

In total 1652 studies were identified from the systematic literature search, of which 373 studies were duplicates. The remaining 1279 unique studies were subjected to first stage screening for eligibility by reading the titles and abstracts, and as a result, 1201 studies were excluded because they were not relevant, and 6 articles were not available as full texts. The full-text screening was done for the remaining 72 articles, of which 48 were included (Fig. 1).

Table 2 shows the summary characteristics of the included studies. Most of the studies used cross-sectional design and data were collected at households and healthcare facilities depending on the chosen costing perspective. Out of the 48 studies, 36 were relatively recent and were published in the year 2010 or after. All studies were of moderate to high quality and provided a good description of the study population and the reason for its selection and the sources used to collect information about resource use. Only a few studies were explicit about the costing methodology used, but the majority provided descriptions of the perspective used.

Figure 2 shows the distribution of the studies in the sub-Saharan African region. The majority were from West and East Africa, while a few were from Southern Africa. 

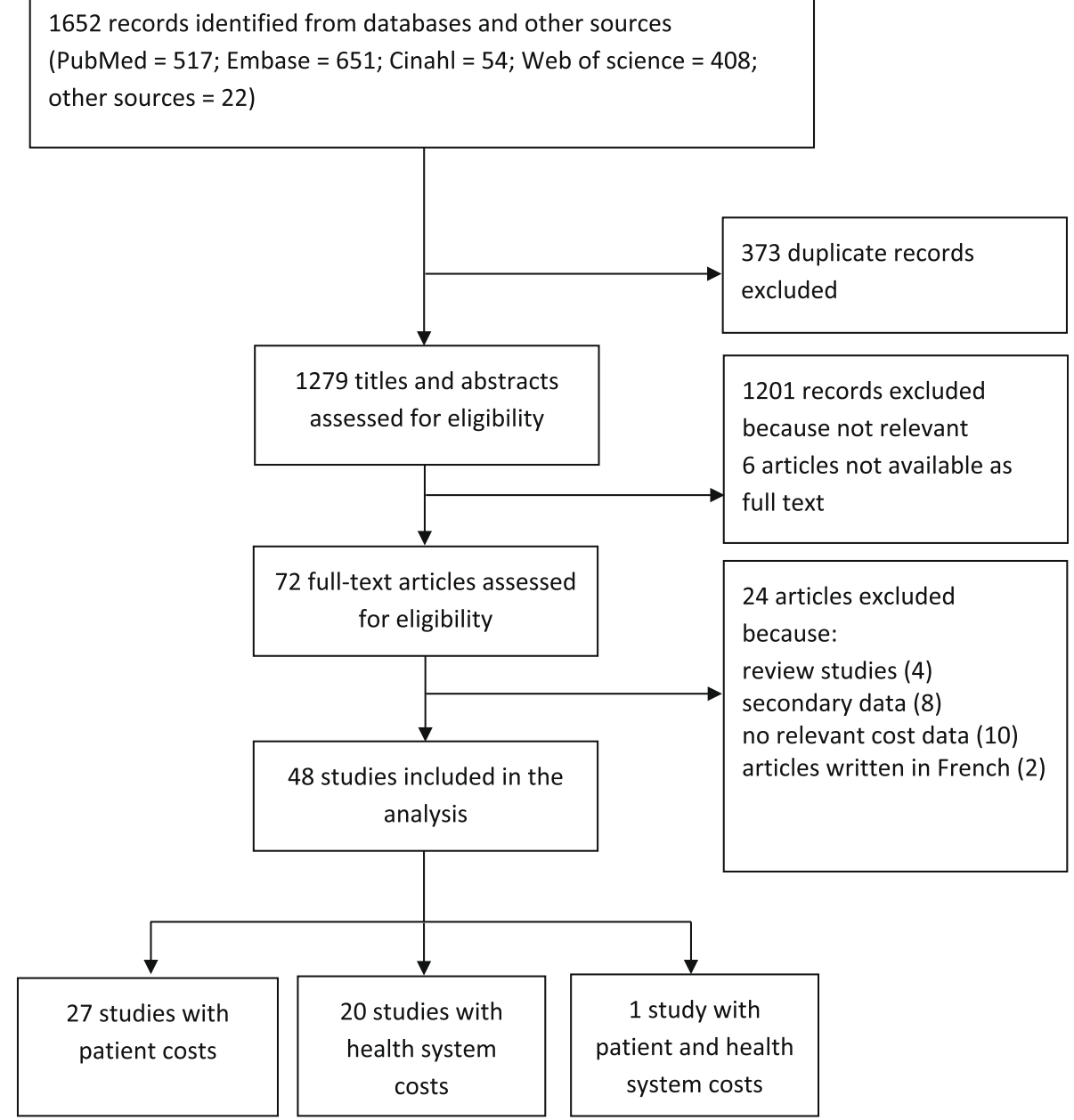

Fig. 1 Flow diagram showing articles included and excluded in the systematic review

Table 3 shows the unit costs for normal delivery and C-section services. There were 19 studies from 27 countries that reported the costs of normal delivery and 20 studies from 24 countries that reported the costs of Csection. Patient cost $(n=13)$ for normal delivery range from USD 5.6-52.4 and the health system cost $(n=6)$ range from USD 8.4-72.8. However, only five of the thirteen studies reported both direct and indirect patient costs and four of the six studies reported both recurrent and capital health system costs. The patient cost $(n=11)$ for C-section ranges from USD 55.8-377.3 but only three of the eleven studies reported both direct and indirect patient costs. The health system cost $(n=9)$ for C-section ranges from USD 79.7-561.8 but only seven of the nine studies reported both recurrent and capital health system costs.

Table 4 shows the unit costs for abortion and postabortion care services (PAC). There were 9 studies from 8 countries that reported the costs of abortion and 4 that reported the costs of PAC. Cost of abortion care represented mostly the medical abortion, while costs of PAC represented unsafe abortions (complete or incomplete), often performed outside the hospital setting with the woman ending up in hospital as a result of complications. Patient cost $(n=8)$ and health system costs for abortion care services $(n=4)$ range from USD 11.2-65.7 and USD 40.3-298.3, respectively. Only two of the eight studies reported direct and indirect patient costs while three of the four studies reported both recurrent and capital health system costs. For PAC services the reported patient cost $(n=6)$ ranges from USD 20.8-158.4 and all studies reported direct costs only. The health system costs for PAC $(n=8)$ were in the range between USD 46.1-151.1, and three of the eight studies reported both recurrent and capital costs.

Table 5 shows the unit costs for the management of eclampsia, low birth weight, and hemorrhage. There were 17 unique studies conducted in 22 countries. For the management of eclampsia, patient costs $(n=5)$ range between USD 51.6-230.5, and two of the five studies 
Table 2 Study characteristics

\begin{tabular}{|c|c|c|c|c|c|c|}
\hline Author and year & Country & Setting & Study design & Target population & $\begin{array}{l}\text { Year of data } \\
\text { collection }\end{array}$ & $\begin{array}{l}\text { Quality } \\
\text { assessment }\end{array}$ \\
\hline Adamu et al. (2012) [16] & Nigeria & Urban & $\begin{array}{l}\text { Cross-sectional } \\
\text { facility based }\end{array}$ & $\begin{array}{l}\text { Surviving women admitted } \\
\text { for obstetric complications }\end{array}$ & 2011 & High \\
\hline Akalu et al. (2012) [17] & Ethiopia & Largely rural & $\begin{array}{l}\text { Cross-sectional } \\
\text { household survey }\end{array}$ & $\begin{array}{l}\text { Women (15-49 years) who } \\
\text { have used reproductive } \\
\text { health services in the past } \\
12 \text { months }\end{array}$ & $2007-2008$ & High \\
\hline Arsenault et al. (2013) [18] & Mali & Urban \& Rural & $\begin{array}{l}\text { Case-control and } \\
\text { household survey }\end{array}$ & $\begin{array}{l}\text { Women with obstetric } \\
\text { emergencies }\end{array}$ & 2008-2011 & Moderate \\
\hline Asante et al. (2007) [19] & Ghana & Unspecified & Facility survey & $\begin{array}{l}\text { Women who had vaginal } \\
\text { deliveries at health facilities, } \\
\text { at homes and those who } \\
\text { had C-sections }\end{array}$ & 2004-2005 & High \\
\hline Benson et al. (2015) [20] & Malawi & Urban \& Rural & $\begin{array}{l}\text { Cross-sectional } \\
\text { facility survey }\end{array}$ & $\begin{array}{l}\text { Women with unsafe } \\
\text { abortion complications }\end{array}$ & 2010 & High \\
\hline \multirow[t]{2}{*}{ Borghi et al. (2003) [21] } & Benin & Unspecified & $\begin{array}{l}\text { Cross-sectional } \\
\text { hospital-based }\end{array}$ & $\begin{array}{l}\text { Women with spontaneous } \\
\text { vaginal delivery and near- } \\
\text { miss obstetric complications }\end{array}$ & 2000 & High \\
\hline & Ghana & Unspecified & $\begin{array}{l}\text { Cross-sectional } \\
\text { hospital-based }\end{array}$ & $\begin{array}{l}\text { Women with spontaneous } \\
\text { vaginal delivery and near- } \\
\text { miss obstetric complications }\end{array}$ & 1999-2000 & High \\
\hline Both et al. (2007) [22] & Tanzania & Urban & $\begin{array}{l}\text { Cross-sectional } \\
\text { hospital-based }\end{array}$ & $\begin{array}{l}\text { Women receiving maternal } \\
\text { healthcare services }\end{array}$ & 2007 & High \\
\hline Carnelissen et al. (2017) [23] & Malawi & Unspecified & $\begin{array}{l}\text { Cross-sectional } \\
\text { hospital-based }\end{array}$ & $\begin{array}{l}\text { Patients including women } \\
\text { requiring a surgical procedure }\end{array}$ & 2014-2015 & High \\
\hline Dalaba et al. (2013) [24] & Ghana & Unspecified & $\begin{array}{l}\text { Cross-sectional } \\
\text { hospital-based }\end{array}$ & $\begin{array}{l}\text { Women receiving antenatal } \\
\text { and delivery services }\end{array}$ & 2010 & High \\
\hline Dalaba et al. (2015) [25] & Ghana & Unspecified & $\begin{array}{l}\text { Cross-sectional } \\
\text { household survey }\end{array}$ & $\begin{array}{l}\text { Women with pregnancy- } \\
\text { related complications }\end{array}$ & 2014 & High \\
\hline Deboutte et al. (2013) [26] & DR Congo & Unspecified & $\begin{array}{l}\text { Cross-sectional } \\
\text { hospital based }\end{array}$ & $\begin{array}{l}\text { Women seeking pregnancy } \\
\text { and obstetric care }\end{array}$ & $2007-2008$ & Moderate \\
\hline Deboutte et al. (2015) [27] & DR Congo & Urban and Rural & Case-Control & $\begin{array}{l}\text { Women with Caesarean section } \\
\text { and vaginal delivery in public } \\
\text { facilities }\end{array}$ & $2007-2008$ & Moderate \\
\hline $\begin{array}{l}\text { Enweronu-Laryea et al. } \\
\text { (2018) [28] }\end{array}$ & Ghana & Urban & $\begin{array}{l}\text { Cross-sectional } \\
\text { hospital-based }\end{array}$ & $\begin{array}{l}\text { Newborns hospitalized with } \\
\text { birth-associated brain injury } \\
\text { and preterm/low birth weight }\end{array}$ & 2016 & High \\
\hline Henshaw et al. (2008) [29] & Nigeria & Urban \&Rural & $\begin{array}{l}\text { Cross-sectional } \\
\text { hospital-based }\end{array}$ & $\begin{array}{l}\text { Women admitted to hospital } \\
\text { for complications of induced } \\
\text { or spontaneous abortion or to } \\
\text { obtain an abortion }\end{array}$ & $2002-2003$ & Moderate \\
\hline Honda et al. (2011) [30] & Madagascar & Mainly urban & $\begin{array}{l}\text { Cross-sectional } \\
\text { hospital-based }\end{array}$ & $\begin{array}{l}\text { Women having C-sections and } \\
\text { children admitted for neonatal } \\
\text { care }\end{array}$ & $2007-2008$ & High \\
\hline Ilboudo et al. (2015) [31] & Burkina Faso & Urban & $\begin{array}{l}\text { Cross-sectional } \\
\text { hospital-based }\end{array}$ & $\begin{array}{l}\text { Women with induced or } \\
\text { spontaneous abortions }\end{array}$ & 2012 & High \\
\hline Ilboudo et al. (2016) [32] & Burkina Faso & Urban & $\begin{array}{l}\text { Cross-sectional } \\
\text { hospital-based }\end{array}$ & $\begin{array}{l}\text { Women with induced abortion } \\
\text { complications }\end{array}$ & 2010 & High \\
\hline Johns et al. (2019) [33] & $\begin{array}{l}\text { Uganda } \\
\text { Zambia }\end{array}$ & Unspecified & Retrospective & $\begin{array}{l}\text { Women attending health } \\
\text { facilities for maternal and } \\
\text { newborn healthcare services }\end{array}$ & 2017-2018 & High \\
\hline Kalu-Umeh et al. (2013) [34] & Nigeria & Semi-rural & $\begin{array}{l}\text { Cross-sectional } \\
\text { community based }\end{array}$ & $\begin{array}{l}\text { Women within the reproductive } \\
\text { age group who had experienced } \\
\text { childbirth } 12 \text { months or less } \\
\text { before the study. }\end{array}$ & 2010 & Moderate \\
\hline $\begin{array}{l}\text { Kowalewski et al. } \\
\text { (2002) [35] }\end{array}$ & Tanzania & Urban and Rural & $\begin{array}{l}\text { Cross-sectional } \\
\text { hospital-based }\end{array}$ & $\begin{array}{l}\text { Women receiving antenatal and } \\
\text { maternal healthcare services }\end{array}$ & 1997-1998 & High \\
\hline
\end{tabular}


Table 2 Study characteristics (Continued)

\begin{tabular}{|c|c|c|c|c|c|c|}
\hline Author and year & Country & Setting & Study design & Target population & $\begin{array}{l}\text { Year of data } \\
\text { collection }\end{array}$ & $\begin{array}{l}\text { Quality } \\
\text { assessment }\end{array}$ \\
\hline Kruk et al. (2008) [36] & Tanzania & Rural & Retrospective & $\begin{array}{l}\text { Women who delivered in } \\
\text { health facilities within the } \\
\text { previous } 5 \text { year }\end{array}$ & 2007 & Moderate \\
\hline Le et al. (2015) [37] & South Africa & Unspecified & $\begin{array}{l}\text { Cross-sectional } \\
\text { hospital-based }\end{array}$ & $\begin{array}{l}\text { Women with unintended } \\
\text { pregnancies }\end{array}$ & 2014 & Moderate \\
\hline Levin et al. (2000) [38] & $\begin{array}{l}\text { Uganda } \\
\text { Malawi } \\
\text { Ghana }\end{array}$ & Unspecified & $\begin{array}{l}\text { Cross-sectional } \\
\text { hospital-based }\end{array}$ & $\begin{array}{l}\text { Women presenting in } \\
\text { healthcare facilities for } \\
\text { maternal health services }\end{array}$ & 1998 & Moderate \\
\hline Lince et al. (2015) [39] & South Africa & Urban & $\begin{array}{l}\text { Cross-sectional } \\
\text { hospital-based }\end{array}$ & $\begin{array}{l}\text { Women accessing } 2 \text { nd } \\
\text { trimester abortion services }\end{array}$ & 2010 & High \\
\hline Lince et al. (2018) [40] & South Africa & Urban & $\begin{array}{l}\text { Cross-sectional } \\
\text { hospital based }\end{array}$ & $\begin{array}{l}\text { Women accessing } 2 \text { nd } \\
\text { trimester abortion services }\end{array}$ & 2013-2014 & High \\
\hline Lince et al. (2017) [41] & South Africa & Urban & $\begin{array}{l}\text { Cross-sectional } \\
\text { hospital based }\end{array}$ & $\begin{array}{l}\text { Women accessing } 1 \text { st } \\
\text { trimester abortion services }\end{array}$ & 2009-2011 & High \\
\hline Lince et al. (2017) [42] & South Africa & Urban & $\begin{array}{l}\text { Cross-sectional } \\
\text { hospital-based }\end{array}$ & $\begin{array}{l}\text { Women accessing 1st } \\
\text { trimester abortion services }\end{array}$ & $2011-2013$ & High \\
\hline Lofgren et al. (2015) [43] & Uganda & $\begin{array}{l}\text { Rural/Semi- } \\
\text { urban }\end{array}$ & Prospective observational & $\begin{array}{l}\text { Patients including women } \\
\text { requiring a surgical procedure }\end{array}$ & 2011 & High \\
\hline Meda et al. (2019) [44] & Burkina Faso & $\begin{array}{l}\text { Urban and } \\
\text { Rural }\end{array}$ & $\begin{array}{l}\text { Cross-sectional } \\
\text { hospital-based }\end{array}$ & $\begin{array}{l}\text { Women who had delivered } \\
\text { or received emergency } \\
\text { obstetric care at public } \\
\text { health facilities }\end{array}$ & 2016 & High \\
\hline Ministry of Health [45] & Kenya & $\begin{array}{l}\text { Urban and } \\
\text { Rural }\end{array}$ & $\begin{array}{l}\text { Cross-sectional } \\
\text { facility survey }\end{array}$ & $\begin{array}{l}\text { Women treated for unsafe } \\
\text { abortion complications }\end{array}$ & 2016 & High \\
\hline Moore et al. (2018) [46] & Zambia & Urban & $\begin{array}{l}\text { Cross-sectional } \\
\text { hospital-based }\end{array}$ & $\begin{array}{l}\text { Women receiving safe and } \\
\text { unsafe abortions }\end{array}$ & 2014-2015 & Moderate \\
\hline Ntambue et al. (2018) [47] & DRC Congo & Urban & $\begin{array}{l}\text { Cross-sectional } \\
\text { hospital-based }\end{array}$ & $\begin{array}{l}\text { Women receiving services in } \\
\text { maternity wards }\end{array}$ & 2014 & High \\
\hline Odhiambo et al. (2019) [48] & Rwanda & Rural & Retrospective & $\begin{array}{l}\text { Women who delivered by } \\
\text { emergency cesarean section }\end{array}$ & 2015 & High \\
\hline Orach et al. (2007) [49] & Uganda & Rural & $\begin{array}{l}\text { Cross-sectional } \\
\text { hospital-based }\end{array}$ & $\begin{array}{l}\text { Women receiving } \\
\text { reproductive health services }\end{array}$ & 2003 & Moderate \\
\hline Parmar et al. (2017) [50] & Zambia & Urban & $\begin{array}{l}\text { Cross-sectional } \\
\text { hospital-based }\end{array}$ & $\begin{array}{l}\text { Women receiving safe and } \\
\text { unsafe abortions }\end{array}$ & 2013-2014 & Moderate \\
\hline Paul et al. (2015) [51] & Sierra Leone & Urban and Rural & Cross-sectional & $\begin{array}{l}\text { Women with unsafe } \\
\text { abortion complications }\end{array}$ & 2012 & Moderate \\
\hline Pearson et al. (2011) [52] & Ethiopia & Urban and Rural & $\begin{array}{l}\text { Cross-sectional } \\
\text { hospital-based }\end{array}$ & $\begin{array}{l}\text { Women receiving } \\
\text { maternity services }\end{array}$ & 2008-2009 & Moderate \\
\hline Perkins et al. (2009) [53] & $\begin{array}{l}\text { Burkina Faso } \\
\text { Kenya } \\
\text { Tanzania }\end{array}$ & $\begin{array}{l}\text { Predominantly } \\
\text { Rural }\end{array}$ & $\begin{array}{l}\text { Cross-sectional } \\
\text { household survey }\end{array}$ & $\begin{array}{l}\text { Women receiving } \\
\text { maternity services }\end{array}$ & 2006 & Moderate \\
\hline Ravit et al. (2015) [54] & Mali & Unspecified & Case-control & $\begin{array}{l}\text { Women who underwent } \\
\text { Caesarean section }\end{array}$ & 2008-2011 & Moderate \\
\hline Ridde et al. (2012) [55] & Burkina Faso & Rural & $\begin{array}{l}\text { Cross-sectional } \\
\text { household survey }\end{array}$ & $\begin{array}{l}\text { Women with vaginal } \\
\text { (normal) delivery }\end{array}$ & 2010 & Moderate \\
\hline Sambo et al. (2013) [56] & Nigeria & Rural & $\begin{array}{l}\text { Cross-sectional } \\
\text { household survey }\end{array}$ & $\begin{array}{l}\text { Pregnant women and those } \\
\text { who delivered recently } \\
\text { (within } 6 \text { weeks postpartum) }\end{array}$ & 2011 & High \\
\hline Sicuri et al. (2011) [57] & Mozambique & Rural & $\begin{array}{l}\text { Cross-sectional } \\
\text { hospital-based }\end{array}$ & Low birth weight babies & 2007 & High \\
\hline Sundaram et al. (2013) [58] & Uganda & Urban and Rural & $\begin{array}{l}\text { Cross-sectional } \\
\text { household survey }\end{array}$ & $\begin{array}{l}\text { Women who received } \\
\text { post-abortion care }\end{array}$ & 2011-2012 & High \\
\hline
\end{tabular}


Table 2 Study characteristics (Continued)

\begin{tabular}{|c|c|c|c|c|c|c|}
\hline Author and year & Country & Setting & Study design & Target population & $\begin{array}{l}\text { Year of data } \\
\text { collection }\end{array}$ & $\begin{array}{l}\text { Quality } \\
\text { assessment }\end{array}$ \\
\hline Tongo et al. (2009) [59] & Nigeria & Urban & $\begin{array}{l}\text { Cross-sectional } \\
\text { hospital-based }\end{array}$ & $\begin{array}{l}\text { Pre term/Low birth } \\
\text { weight neonates }\end{array}$ & 2008 & High \\
\hline Vlassoff et al. (2012) [60] & Ethiopia & Urban and Rural & $\begin{array}{l}\text { Cross-sectional } \\
\text { hospital-based }\end{array}$ & $\begin{array}{l}\text { Women who received } \\
\text { post-abortion care }\end{array}$ & 2008 & High \\
\hline Vlassoff et al. (2014) [61] & Uganda & Urban and Rural & $\begin{array}{l}\text { Cross-sectional } \\
\text { hospital-based }\end{array}$ & $\begin{array}{l}\text { Women who received } \\
\text { post-abortion care }\end{array}$ & 2010 & High \\
\hline Vlassoff et al. (2015) [62] & Rwanda & Urban and Rural & $\begin{array}{l}\text { Cross-sectional } \\
\text { hospital-based }\end{array}$ & $\begin{array}{l}\text { Women who received } \\
\text { post-abortion care }\end{array}$ & 2012 & High \\
\hline Witter et al. (2010) [63] & Senegal & Urban and Rural & $\begin{array}{l}\text { Cross-sectional } \\
\text { hospital-based }\end{array}$ & $\begin{array}{l}\text { Women receiving } \\
\text { Caesarean section and } \\
\text { those with normal delivery }\end{array}$ & $2006-2007$ & Moderate \\
\hline
\end{tabular}

reported both direct and indirect costs. The health system costs for eclampsia $(n=2)$ range from USD 122.7-186.4 and no capital cost was measured. For care of low-birth weight babies, the patient cost $(n=3)$ ranged between USD 38.2-486.7, and two studies contained both direct and indirect costs, while only one study by Sicuri et al. (2011) from Mozambique reported an average health system cost of about USD 514 for caring such babies. For the management of maternal hemorrhage, patient cost $(n=4)$ ranges between USD 65.1-196.2, and half of the studies reported both direct and indirect costs. The health system cost for maternal hemorrhage range between USD 30.3-127.4 and all the studies reported recurrent health system cost only.

Figures 3 and 4 compare whether out of pocket health expenditures for normal delivery, C-section, eclampsia, and maternal hemorrhage was higher than $10 \%$ of the average gross national income per capita for different countries in sub-Saharan Africa. Out of pocket cost for normal delivery services was catastrophic for only one study from DR Congo

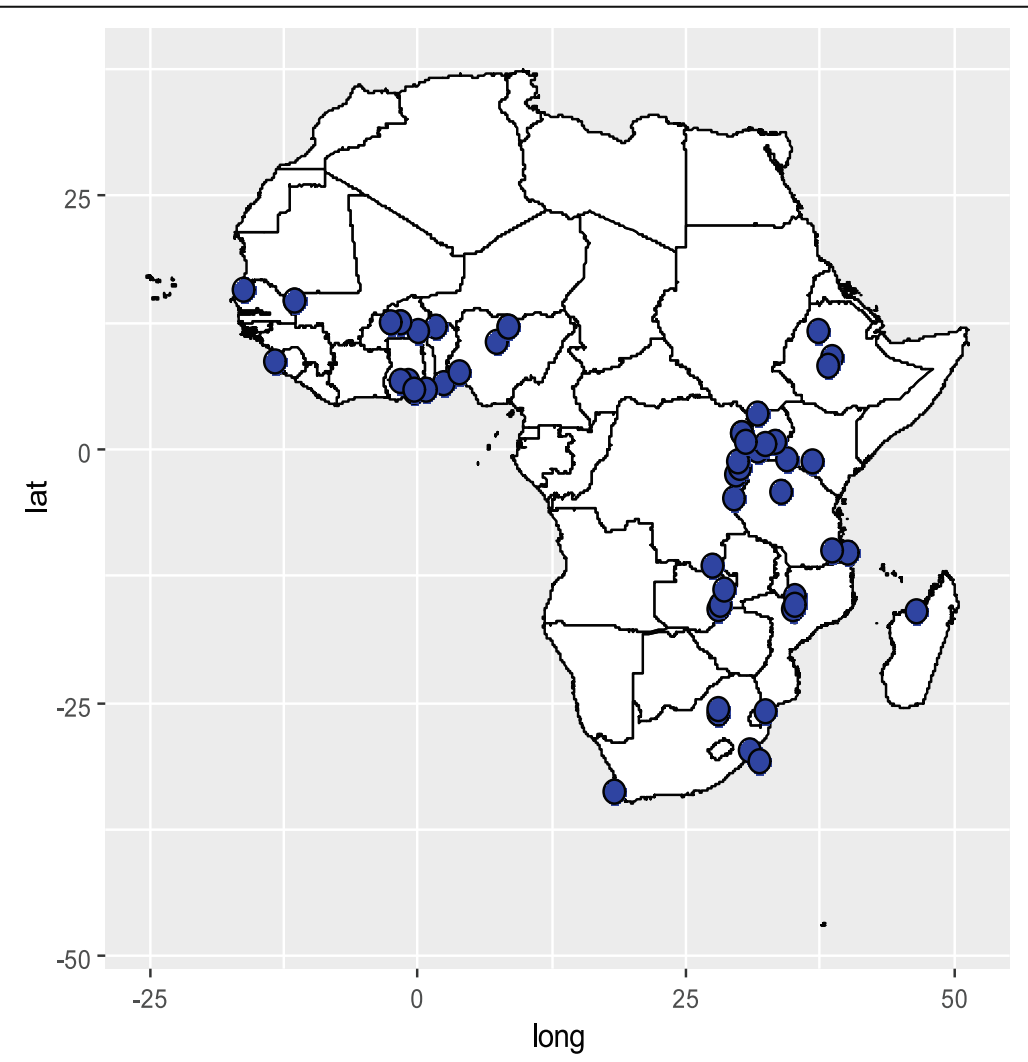

Fig. 2 Distribution of studies in sub-Saharan Africa 
Table 3 Costs for normal delivery and Caesarean sections

\begin{tabular}{|c|c|c|c|c|c|}
\hline Authors name & Country & Data collection year & Cost category & Base year cost (USD) & Cost (USD) in 2018 \\
\hline \multicolumn{6}{|l|}{ Normal delivery } \\
\hline \multicolumn{6}{|l|}{ Patient perspective } \\
\hline Asante et al. (2007) [19] & Ghana & 2004 & Direct & 42.1 & 52.4 \\
\hline \multirow[t]{2}{*}{ Borghi et al. (2003) [21] } & Benin & 2000 & Direct & 23.0 & 33.5 \\
\hline & Ghana & 1999-2000 & Direct & 15.0 & 21.9 \\
\hline Deboutte et al. (2015) [27] & DR Congo & $2007-2008$ & Direct & 15.3 & 18.5 \\
\hline Kalu-Umeh et al. (2013) [34] & Nigeria & 2010 & Direct & 9.0 & 10.4 \\
\hline Kowalewski (2002) [35] & Tanzania & 1997-1998 & Direct \& indirect & 18.5 & 28.5 \\
\hline Kruk et al. (2008) [36] & Tanzania & 2007 & Direct & 6.9 & 8.6 \\
\hline \multirow[t]{3}{*}{ Levin et al. (2000) [38] } & Uganda & 1998 & Direct \& indirect & 17.0 & 26.2 \\
\hline & Malawi & 1998 & Direct \& indirect & 7.8 & 120 \\
\hline & Ghana & 1998 & Direct \& indirect & 16.6 & 25.5 \\
\hline Meda et al. (2019) [44] & Burkina Faso & 2016 & Direct & 6.1 & 6.4 \\
\hline Ntambue et al. (2018) [47] & DR Congo & 2014 & Direct \& indirect & 45.0 & 50.2 \\
\hline \multirow[t]{3}{*}{ Pearson et al. (2011) [52] } & Ethiopia & 2008-2009 & Direct & 14.4 & 16.8 \\
\hline & Tanzania & 2006 & Direct & 4.5 & 5.6 \\
\hline & Burkina Faso & 2006 & Direct & 6.6 & 8.2 \\
\hline Perkins et al. (2009) [53] & Kenya & 2006 & Direct & 14.2 & 17.7 \\
\hline Ridde et al. (2012) [55] & Burkina Faso & 2010 & Direct & 9.9 & 11.4 \\
\hline Sambo et al. (2013) [56] & Nigeria & 2013 & Direct & 9.6 & 10.7 \\
\hline \multicolumn{6}{|l|}{ Provider perspective } \\
\hline Both et al. (2007) [22] & Tanzania & 2003 & Recurrent \& capital & 6.3 & 8.6 \\
\hline Dalaba et al. (2013) [24] & Ghana & 2010 & Recurrent \& capital & 63.2 & 72.8 \\
\hline \multirow[t]{2}{*}{ Johns et al. (2019) [33] } & Uganda & 2017-2018 & Recurrent \& capital & 41.3 & 43.2 \\
\hline & Zambia & 2017-2018 & Recurrent \& capital & 23.0 & 24.1 \\
\hline \multirow[t]{3}{*}{ Levin at al (2000) [38] } & Uganda & 1998 & Recurrent & 21.2 & 32.7 \\
\hline & Malawi & 1998 & Recurrent & 14.3 & 22.0 \\
\hline & Ghana & 1998 & Recurrent & 10.8 & 16.7 \\
\hline Orach et al. (2007) [49] & Uganda & 2003 & Recurrent \& capital & 6.1 & 8.4 \\
\hline Witter et al. (2010) [63] & Senegal & $2006-2007$ & Recurrent & 15.0 & 18.7 \\
\hline \multicolumn{6}{|l|}{ C-section } \\
\hline \multicolumn{6}{|l|}{ Patient perspective } \\
\hline Arsenault et al. (2013) [18] & Mali & 2008-2011 & Direct & $107.0^{*}$ & 119.5 \\
\hline Asante et al. (2007) [19] & Ghana & 2004 & Direct & 195.0 & 242.9 \\
\hline Deboutte et al. (2015) [27] & DR Congo & 2007-2008 & Direct & 79.7 & 96.5 \\
\hline Honda et al. (2011) [30] & Madagascar & $2007-2008$ & Direct & 139.0 & 162.1 \\
\hline Kalu-Umeh et al. (2013) [34] & Nigeria & 2010 & Direct & 99.0 & 114.0 \\
\hline Kowalewski [35] & Tanzania & 1997-1998 & Direct \& indirect & 135.0 & 208.0 \\
\hline \multirow[t]{2}{*}{ Levin et al. (2000) [38] } & Uganda & 1998 & Direct \& indirect & 36.2 & 55.8 \\
\hline & Ghana & 1998 & Direct \& indirect & 104.0 & 160.2 \\
\hline Meda et al. (2019) [44] & Burkina Faso & 2016 & Direct & 136.4 & 142.7 \\
\hline Ntambue et al. (2018) [47] & DR Congo & 2014 & Direct \& indirect & 338.0 & 377.3 \\
\hline Pearson et al. (2011) [52] & Ethiopia & 2008-2009 & Direct & 51.1 & 59.6 \\
\hline Ravit et al. (2015) [54] & Mali & 2008-2011 & Direct & 163.0 & 182.0 \\
\hline
\end{tabular}


Table 3 Costs for normal delivery and Caesarean sections (Continued)

\begin{tabular}{|c|c|c|c|c|c|}
\hline Authors name & Country & Data collection year & Cost category & Base year cost (USD) & Cost (USD) in 2018 \\
\hline \multicolumn{6}{|l|}{ Provider perspective } \\
\hline Both et al. (2007) [22] & Tanzania & 2003 & Recurrent \& capital & 69.3 & 94.5 \\
\hline Cornelissen et al. (2017) [23] & Malawi & 2014-2015 & Recurrent \& capital & 351.0 & 391.8 \\
\hline Deboutte et al. (2013) [26] & DR Congo & 2007-2008 & Recurrent \& capital & 157.8 & 184.0 \\
\hline \multirow[t]{2}{*}{ Johns et al. (2019) [33] } & Uganda & $2017-2018$ & Recurrent \& capital & 238.5 & 249.5 \\
\hline & Zambia & 2017-2018 & Recurrent \& capital & 537 & 561.8 \\
\hline \multirow[t]{3}{*}{ Levin et al. (2000) [38] } & Uganda & 1998 & Recurrent & 79.8 & 122.9 \\
\hline & Malawi & 1998 & Recurrent & 81.9 & 126.1 \\
\hline & Ghana & 1998 & Recurrent & 72.2 & 111.2 \\
\hline Lofgren et al. (2015) [43] & Uganda & 2011 & Recurrent \& capital & 71.4 & 79.7 \\
\hline Odhiambo et al. (2019) [48] & Rwanda & 2015 & Recurrent \& capital & 339 & 359.2 \\
\hline Orach et al. (2007) [49] & Uganda & 2003 & Recurrent \& capital & 58.7 & 80.1 \\
\hline Witter et al. (2010) [63] & Senegal & 2006-2007 & Recurrent & 137.0 & 165.9 \\
\hline
\end{tabular}

${ }^{*}$ represents costs of treatment only

[47], however, costs were catastrophic in eight studies out of the twelve $[18,19,27,30,35,44,47,54]$ that reported delivery by the $\mathrm{C}$-section. Out of pocket payments were also catastrophic in three out of six studies on the management of eclampsia [21, $47,54]$, one out of four studies about abortion services [31], one out of three studies on the management of low birth weight babies [59] and four out of five studies on the management of hemorrhage $[21,44,47,54]$. None of the studies on PAC costs indicated that catastrophic health expenditures were incurred.

\section{Discussion}

This review shows that pregnancy and childbearing expose women and their families to a lot of out-ofpocket (OOP) payments, particularly in the presence of complications. For normal spontaneous vaginal deliveries, women pay between USD 5.6-52.4 and for C-section they pay between USD 55.8-377.3, meaning on average it costs seven times more to deliver by $\mathrm{C}$ section. The OOP payments usually constitute costs of drugs and medical supplies like cotton wools, syringes, transportation to and from the health facilities, food, drinks and unofficial payments to health workers. Mean OOP payments were either very close to or exceeded $10 \%$ of an average national per capita income for some countries, thus most likely exposing patients and their families to substantial financial burden.

Results from the included studies show that catastrophic health expenditures were common among the study participants [16-18, 27, 30, 31, 59]. In Bunia DR Congo, the user cost of C-section was estimated at 79.7 USD, which was slightly above the monthly family income of 75.5 USD [27]. In Birnin-Kebbi Nigeria, average monthly family income was 18.8 USD compared to the average cost of care for emergency obstetric care (EmOC) of about 246 USD [16]. In rural Ethiopia, more than two-thirds of the studied families experienced catastrophic health expenditure for maternal healthcare [17]. In Mali, between 20 and $54 \%$ of the studies households incurred catastrophic health expenditure on EmOC [18]. In Burkina Faso, 12\% of women with abortion experienced catastrophic health expenditure [31]. In Madagascar, the proportion of OOP for Csection among the richest and the poor was $33 \%$ and $109 \%$, respectively [30].

Our study indicates that pregnancy and childbearing complications are also relatively expensive to the healthcare systems in sub-Saharan Africa. Health systems use between USD 8-73 per patient for normal deliveries, but a staggering USD 80-562 for Csection, USD 40-300 for medical abortion, USD 40150 for post-abortion care, USD 120-190 to care for eclampsia, USD 30-130 to treat hemorrhage and about USD 500 to care for low-birth weight babies. In 2009 it was estimated that the annual cost to treat unsafe abortion complications in sub-Saharan Africa ranged from USD 68-76 million [64] and in 2014, it was estimated that the cost required to provide postabortion care in developing countries was USD 232 million [65]. A large chunk of these costs could be prevented by investing in modern contraceptive use to prevent unwanted pregnancies, legalizing abortion where it is illegal and implementing policies with the potential to reduce adolescent pregnancies. High costs that are associated with access to healthcare hinder the utilization of maternal health services in resourcepoor settings [66, 67]. 
Table 4 Costs for abortion and PAC

\begin{tabular}{|c|c|c|c|c|c|}
\hline Authors name & Country & Data collection year & Cost category & Base cost (USD) & Cost (USD) in 2018 \\
\hline \multicolumn{6}{|l|}{ Abortion } \\
\hline \multicolumn{6}{|l|}{ Patient perspective } \\
\hline Akalu et al. (2012) [17] & Ethiopia & 2007-2008 & Direct & $13.4^{\mathrm{a}}$ & 15.7 \\
\hline Henshaw et al. (2008) [29] & Nigeria & 2002-2003 & Direct & 32.2 & 43.9 \\
\hline \multirow[t]{2}{*}{ Ilboudo et al. (2015) [31] } & Burkina Faso & 2012 & Direct & $56.0^{\mathrm{b}}$ & 61.3 \\
\hline & Burkina Faso & 2012 & Direct & $37.0^{c}$ & 40.5 \\
\hline Lince et al. (2015) [39] & South Africa & 2010 & Direct \& indirect & 21.2 & 24.5 \\
\hline Lince et al. (2017) [41, 42] & South Africa & 2009-2011 & Direct \& indirect & 10.0 & 11.2 \\
\hline Moore et al. (2018) [46] & Zambia & 2014-2015 & Direct & 62.0 & 65.7 \\
\hline Pearson et al. (2011) [52] & Ethiopia & 2008-2009 & Direct & 10.0 & 11.7 \\
\hline Sundaram et al. (2013) [58] & Uganda & 2011-2012 & Direct & 23.0 & 25.2 \\
\hline \multicolumn{6}{|l|}{ Provider perspective } \\
\hline Le et al. (2015) [37] & South Africa & 2014 & Recurrent \& capital & 281.2 & 298.3 \\
\hline Lince et al. (2017) [41, 42] & South Africa & $2011-2013$ & Recurrent \& capital & 65.4 & 70.5 \\
\hline Lince et al. (2018) [40] & South Africa & 2013-2014 & Recurrent \& capital & $250.3^{d}$ & 265.5 \\
\hline Parmar D [50] & Zambia & 2013-2014 & Recurrent & $38.0^{\mathrm{e}}$ & 40.3 \\
\hline \multicolumn{6}{|l|}{ Post-abortion care } \\
\hline \multicolumn{6}{|l|}{ Patient perspective } \\
\hline Henshaw et al. (2008) [29] & Nigeria & 2002-2003 & Direct & 116.0 & 158.4 \\
\hline \multirow[t]{2}{*}{ Ilboudo et al. (2015) [31] } & Burkina Faso & 2012 & Direct & $33.0^{\mathrm{b}}$ & 36.1 \\
\hline & Burkina Faso & 2012 & Direct & $19.0^{c}$ & 20.8 \\
\hline Meda et al. (2019) [44] & Burkina Faso & 2016 & Direct & 32.1 & 33.6 \\
\hline Moore et al. (2018) [46] & Zambia & 2014-2015 & Direct & 81.0 & 85.8 \\
\hline Sundaram et al. (2013) [58] & Uganda & 2011-2012 & Direct & 26.0 & 28.4 \\
\hline \multicolumn{6}{|l|}{ Provider perspective } \\
\hline Benson et al. (2015) [20] & Malawi & 2010 & Recurrent & 40.0 & 46.1 \\
\hline \multirow[t]{3}{*}{ Levin et al. (2000) [38] } & Uganda & 1998 & Recurrent & 46.5 & 71.7 \\
\hline & Malawi & 1998 & Recurrent & 35.9 & 55.2 \\
\hline & Ghana & 1998 & Recurrent & 65.2 & 100.4 \\
\hline MoH -Kenya (2018) [45] & Kenya & 2016 & Recurrent & 58.0 & 60.7 \\
\hline Parmar et al. (2017) [50] & Zambia & 2013-2014 & Recurrent & 52.0 & 55.2 \\
\hline Paul et al. (2015) [51] & Sierra Leone & 2012 & Recurrent & 68.0 & 74.4 \\
\hline Vlassoff et al. (2014) [61] & Rwanda & 2012 & Recurrent \& capital & 93.0 & 101.7 \\
\hline Vlassoff et al. (2012) [60] & Uganda & 2010 & Recurrent \& capital & 131.2 & 151.1 \\
\hline Vlassoff et al. (2009) [64] & Ethiopia & 2008 & Recurrent \& capital & 100.0 & 116.6 \\
\hline
\end{tabular}

average of costs in public facilities (USD 16.12) and private USD 10.73

b procedure for induced abortion

c cost of care for spontaneous abortion

daverage for dilatation \& evacuation with misoprostol (88.90 USD), medical induction with mifepristone+misoprostol (298.03 USD) and medical induction with misoprostol only (364.08 USD)

${ }^{\mathrm{e}}$ Costs of unsafe abortion

This study has several limitations, which requires care in its interpretation. Firstly, the included studies were methodologically very heterogeneous in terms of range patient and health system costs included making it hard to fully disaggregate the costs. Secondly, costs are very context-specific especially for nontraded goods and services such as wages and salaries, which are usually one of the main cost drivers. Thus, in countries where salaries and prices of commodities are high always tend to skew the average costs. Also, 
Table 5 Costs of other complications

\begin{tabular}{|c|c|c|c|c|c|}
\hline Authors name & Country & Data collection year & Cost category & Base cost (USD) & Cost (USD) in 2018 \\
\hline \multicolumn{6}{|l|}{ Eclampsia } \\
\hline \multicolumn{6}{|l|}{ Patient perspective } \\
\hline \multirow[t]{2}{*}{ Borghi et al. (2003) [21] } & Benin & 2000 & Direct & 119.0 & 173.5 \\
\hline & Ghana & 1999-2000 & Direct & 69.0 & 100.6 \\
\hline Dalaba et al. (2015) [25] & Ghana & 2014 & Direct \& indirect & 58.3 & 61.9 \\
\hline Meda et al. (2019) [44] & Burkina Faso & 2016 & Direct & 49.3 & 51.6 \\
\hline Ntambue et al. (2018) [47] & DR Congo & 2014 & Direct \& indirect & $206.5^{b}$ & 230.5 \\
\hline Ravit et al. (2015) [54] & Mali & 2008-2011 & Direct & 179.8 & 200.7 \\
\hline \multicolumn{6}{|l|}{ Provider perspective } \\
\hline \multirow[t]{2}{*}{ Levin et al. (2000) [38] } & Uganda & 1998 & Recurrent & 121.015 & 186.4 \\
\hline & Malawi & 1998 & Recurrent & 79.62 & 122.7 \\
\hline \multicolumn{6}{|l|}{ Low birth weight babies ${ }^{a}$} \\
\hline \multicolumn{6}{|l|}{ Patient perspective } \\
\hline Enweronu et al. (2018) [28] & Ghana & 2016 & Direct \& indirect & 147.6 & 154.4 \\
\hline Sicuri et al. (2011) [57] & Mozambique & 2007 & Direct \& indirect & 31.5 & 38.2 \\
\hline Tongo et al. (2008) [59] & Nigeria & 2008 & Direct & 417.3 & 486.7 \\
\hline \multicolumn{6}{|l|}{ Provider perspective } \\
\hline Sicuri et al. (2011) [57] & Mozambique & 2007 & Recurrent \& capital & 424.6 & 514.2 \\
\hline \multicolumn{6}{|l|}{ Hemorrhage } \\
\hline \multicolumn{6}{|l|}{ Patient perspective } \\
\hline \multirow[t]{2}{*}{ Borghi et al. (2003) [21] } & Benin & 2000 & Direct & 104.0 & 151.7 \\
\hline & Ghana & 1999-2000 & Direct & 79.0 & 115.2 \\
\hline Dalaba et al. (2015) [25] & Ghana & 2014 & Direct \& indirect & 6.84 & 7.3 \\
\hline Meda et al. (2019) [44] & Burkina Faso & 2016 & Direct & 58.35 & 65.2 \\
\hline Ntambue et al. (2018) [47] & DR Congo & 2014 & Direct \& indirect & $187.5^{b}$ & 196.17 \\
\hline Ravit et al. (2015) [54] & Mali & 2008-2011 & Direct & 140.34 & 156.67 \\
\hline \multicolumn{6}{|l|}{ Provider perspective } \\
\hline Ilboudo et al. (2016) [32] & Burkina Faso & 2010 & Recurrent & 26.3 & 30.3 \\
\hline \multirow[t]{3}{*}{ Levin et al. (2000) [38] } & Uganda & 1998 & Recurrent & 82.7 & 127.4 \\
\hline & Malawi & 1998 & Recurrent & 74.3 & 114.5 \\
\hline & Ghana & 1998 & Recurrent & 65.3 & 100.5 \\
\hline
\end{tabular}

${ }^{a}$ Costs from delivery to discharge from hospital

${ }^{\mathrm{b}}$ Average cost for vaginal and c-section

there could be a lot of variations in the structure and complexity of the healthcare system and services available for managing pregnancy and birth-related complications between countries, hence resource requirements and costs could infinitely vary from one place to another. For this reason, we could not aggregate the costs into meaningful means or medians.

Our findings regarding the costs of maternal health care have several policy implications despite the limitations. First, it is well documented that adolescent pregnancy and childbearing are associated with elevated risks of complications $[68,69]$, which are mainly concentrated in sub-Saharan Africa [70]. Our study enhances the understanding of the financial implications of these complications both for patients, families, and health systems. Policies that can delay teen pregnancies, therefore, have the potential not only to reduce maternal morbidity and mortality but also to save patients and health systems a significant amount of healthcare resources. Second, this review shows that maternal complications may result in OOP expenditures that are largely catastrophic especially among the poorest households. New innovative strategies are urgently needed to protect women and 

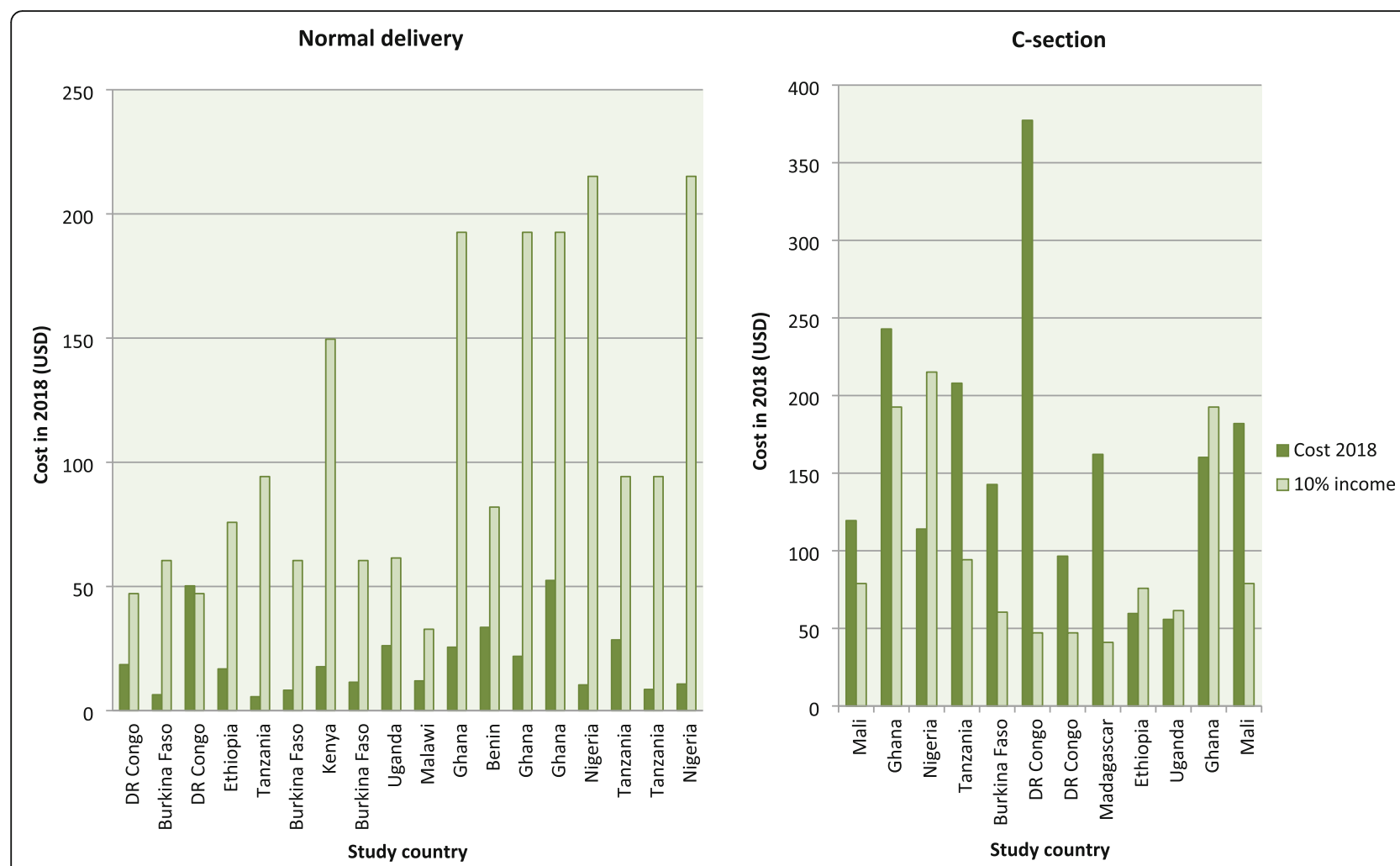

Fig. 3 Out of pocket cost versus 10\% of per capita income for normal vaginal delivery and C-section

their families from impoverishing OOP, otherwise, the real impact of abolishing user fees for maternal services will be hard to be realized.

\section{Conclusion}

This is the first systematic literature review to compile comprehensive up-to-date patient and health system costs of managing pregnancy and birthrelated complications in sub-Saharan Africa. It indicates that these costs are relatively high. It further shows that patient costs were largely catastrophic relative to a $10 \%$ of average national per capita income, thus exposing families to immense financial burden and impoverishment, in particularly poor

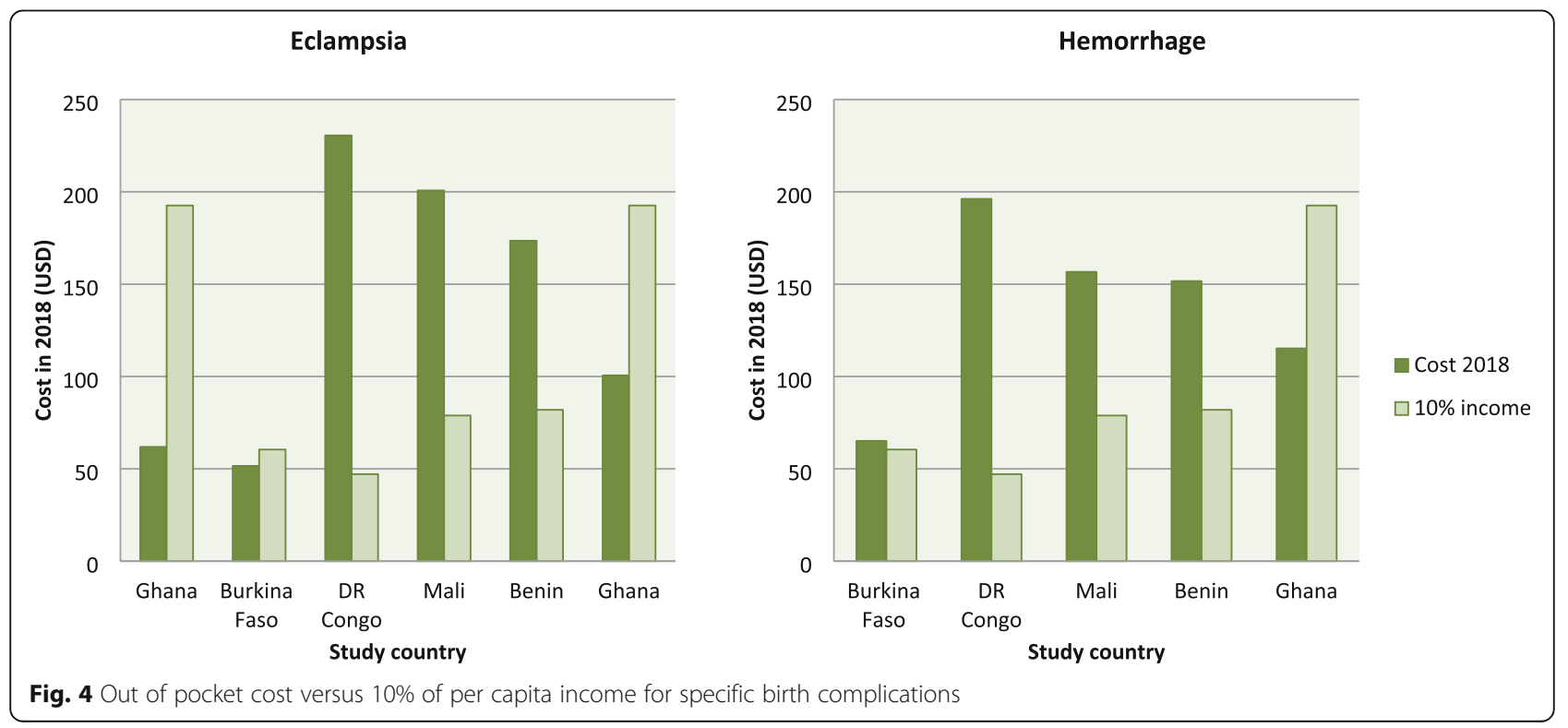


families that live under one USD per day. Hence health policies that advocate for free maternal health services and universal health coverage on maternal and newborn care should be encouraged and prioritized on both national, regional, and international agenda. Otherwise, the high costs will continue to hinder access to maternal health services in subSaharan Africa, thus negating the efforts to reduce infant and maternal mortality rates which are relatively high in this region. Although the study found a relatively large number of studies, the evidence base on the costs of maternal care is nevertheless still scarce; hence, more studies are needed to fill the gaps.

\section{Abbreviations}

C-section: Caesarean Section; EmOC: Emergency obstetric care; GNI: Gross National Income; OOP: Out of pocket; PAC: Post abortion care; USD: United States Dollar

\section{Acknowledgements}

This study is part of the project portfolio of the Center for Intervention Science in Maternal and Child Health (CISMAC), a Center for Excellence (CoE) funded by the Research Council of Norway (RCN) and the University of Bergen. We would like to thank RCN and CISMAC's Scientific Committee for facilitating the conduction of this study.

\section{Authors' contributions}

ATM, BJ and IS contributed to the study conception and design. ATM, PB and PH conducted the search of articles. ATM and PB independently screened the articles. ATM and PH independently performed quality assessment. The first draft of the manuscript was written by ATM and all authors commented on previous versions of the manuscript. All authors read and approved the final manuscript.

\section{Funding}

The study was funded by the Research Council of Norway through its Centers of Excellence scheme to the Center for Intervention Science in Maternal and Child Health (CISMAC; project number 223269) and through the GLOBVAC program (project number 248121). The funder of the study had no role in study design, data collection, data analysis, data interpretation, or writing of the report.

\section{Availability of data and materials}

Not applicable.

\section{Ethics approval and consent to participate}

Not applicable.

\section{Consent for publication}

Not applicable.

\section{Competing interests}

The authors declare that they have no competing interests.

\section{Author details}

${ }^{1}$ Centre for International Health, University of Bergen, P.O. Box 7804, 5020 Bergen, Norway. ${ }^{2}$ Department of Global Public Health and Primary Care, Section for Ethics and Health Economics, University of Bergen, Bergen, Norway. ${ }^{3}$ Centre for Intervention Science in Maternal and Child Health (CISM AC), University of Bergen, Bergen, Norway. ${ }^{4}$ Department of Health System, Impact Evaluation and Policy, Ifakara Health Institute, Dar es Salaam, Tanzania. ${ }^{5}$ Department of Health Policy and Management, School of Public Health, University of Zambia, Lusaka, Zambia.
Received: 4 October 2019 Accepted: 5 August 2020

Published online: 15 August 2020

\section{References}

1. Mombo-Ngoma G, Mackanga JR, Gonzalez R, Ouedraogo S, Kakolwa MA, Manego RZ, Basra A, Ruperez M, Cot M, Kabanywany AM, et al. Young adolescent girls are at high risk for adverse pregnancy outcomes in subSaharan Africa: an observational multicountry study. BMJ Open. 2016;6(6): e011783.

2. Ganchimeg T, Ota E, Morisaki N, Laopaiboon M, Lumbiganon P, Zhang J, Yamdamsuren B, Temmerman M, Say L, Tuncalp O, et al. Pregnancy and childbirth outcomes among adolescent mothers: a World Health Organization multicountry study. BJOG. 2014;121(Suppl 1):40-8.

3. Gronvik T, Fossgard Sandoy I. Complications associated with adolescent childbearing in sub-Saharan Africa: a systematic literature review and metaanalysis. PLoS One. 2018;13(9):e0204327.

4. Mokdad AH, Forouzanfar MH, Daoud F, Mokdad AA, El Bcheraoui C, MoradiLakeh M, Kyu HH, Barber RM, Wagner J, Cercy K, et al. Global burden of diseases, injuries, and risk factors for young people's health during 19902013: a systematic analysis for the global burden of disease study 2013. Lancet. 2016;387(10036):2383-401.

5. Loaiza E, Liang M. Adolescence pregnancy: a review of evidence. New York: United Nations Population Fund; 2013.

6. Huynh L, McCoy M, Law A, Tran KN, Knuth S, Lefebvre P, Sullivan S, Duh MS Systematic literature review of the costs of pregnancy in the US. Pharmacoeconomics. 2013;31(11):1005-30.

7. Petrou S, Eddama O, Mangham L. A structured review of the recent literature on the economic consequences of preterm birth. Arch Dis Child Fetal Neonatal Ed. 2011;96(3):F 225-32.

8. Petrou S, Sach T, Davidson L. The long-term costs of preterm birth and low birth weight: results of a systematic review. Child Care Health Dev. 2001; 27(2):97-115.

9. Mori AT, Kampata L, Musonda P, Johansson KA, Robberstad B, Sandøy I. Cost-benefit and extended cost-effectiveness analysis of a comprehensive adolescent pregnancy prevention program in Zambia: study protocol for a cluster randomized controlled trial. Trials. 2017;18(1):604. https://doi.org/10. 1186/s13063-017-2350-4.

10. Moher D, Liberati A, Tetzlaff J, Altman DG. Preferred reporting items for systematic reviews and meta-analyses: the PRISMA statement. PLoS Med. 2009;6(7):e1000097.

11. Drummond MF, Sculpher MJ, Torrance GW, O'Brien BJ, Stoddart GL. Methods for the economic evaluation of health care programmes. 3rd ed. New York: Oxford University Press; 2005.

12. van Lier LI, Bosmans JE, van Hout HPJ, Mokkink LB, van den Hout WB, de Wit GA, Dirksen CD, Nies H, Hertogh C, van der Roest HG. Consensus-based cross-European recommendations for the identification, measurement and valuation of costs in health economic evaluations: a European Delphi study. Eur J Health Econ. 2018;19(7):993-1008.

13. Husereau D, Drummond M, Petrou S, Carswell C, Moher D, Greenberg D, Augustovski F, Briggs AH, Mauskopf J, Loder E, et al. Consolidated health economic evaluation reporting standards (CHEERS) statement. Cost Effectiveness Resour Allocation: C/E. 2013;11(1):6.

14. IMF Data Access to Macroeconomic and Financial Data. International Monetary Fund: Washington D.C.; 2019.

15. Wagstaff $A$, van Doorslaer $E$. Catastrophe and impoverishment in paying for health care: with applications to Vietnam 1993-1998. Health Econ. 2003;12:921-33.

16. Adamu AN, Adamu H, Yabagi Al, Sa'Ad Z. Expenditure on emergency obstetric care in a tertiary health centre in northern Nigeria. Int J Gynecol Obstet. 2012;3:S264-5.

17. Akalu T, Guda A, Tamiru M, Marian DH. Examining out of pocket payments for maternal health in rural Ethiopia: paradox of free health care unaffordability. Ethiop J Health Dev. 2012;26:251-7.

18. Arsenault C, Fournier P, Philibert A, Sissoko K, Coulibaly A, Tourigny C, Traore M, Dumont A. Emergency obstetric care in Mali: catastrophic spending and its impoverishing effects on households. Bull World Health Organ. 2013;91(3):207-16.

19. Asante F, Chikwama C, Daniels A, Armar-Klemesu M. Evaluating the economic outcomes of the policy of fee exemption for maternal delivery care in Ghana. Ghana Med J. 2007:41(3):110-7. 
20. Benson J, Gebreselassie H, Manibo MA, Raisanen K, Johnston HB, Mhango C, Levandowski BA. Costs of postabortion care in public sector health facilities in Malawi: a cross-sectional survey. BMC Health Serv Res. 2015;15:562.

21. Borghi J, Hanson K, Acquah CA, Ekanmian G, Filippi V, Ronsmans C, Brugha R, Browne E, Alihonou E. Costs of near-miss obstetric complications for women and their families in Benin and Ghana. Health Policy Plan. 2003; 18(4):383-90.

22. von Both C, Jahn A, Fleba S. Costing maternal health services in South Tanzania: a case study from Mtwara Urban District. Eur J Health Econ. 2008; 9(2):103-15.

23. Cornelissen D, Mwapasa G, Gajewski J, et al. The Cost of Providing DistrictLevel Surgery in Malawi. World J Surg. 2018;42(1):46-53. https://doi.org/10. 1007/s00268-017-4166-5.

24. Dalaba MA, Akweongo P, Savadogo G, Saronga H, Williams J, Sauerborn R, Dong $\mathrm{H}$, Loukanova $\mathrm{S}$. Cost of maternal health services in selected primary care centres in Ghana: a step down allocation approach. BMC Health Serv Res. 2013;13:287.

25. Dalaba MA, Akweongo P, Aborigo RA, Saronga HP, Williams J, Aninanya GA, Sauerborn R, Loukanova S. Cost to households in treating maternal complications in northern Ghana: a cross sectional study. BMC Health Serv Res. 2015:15:34.

26. Deboutte D, O'Dempsey T, Mann G, Faragher B. Cost-effectiveness of caesarean sections in a post-conflict environment: a case study of Bunia, Democratic Republic of the Congo. Disasters. 2013;37(Suppl 1):S105-20.

27. Deboutte D, O'Dempsey T, Mann G, Faragher B. User cost of caesarean section: case study of Bunia, Democratic Republic of Congo. Int J Health Plann Manag. 2015;30(2):88-97.

28. Enweronu-Laryea CC, Andoh HD, Frimpong-Barfi A, Asenso-Boadi FM. Parental costs for in-patient neonatal services for perinatal asphyxia and low birth weight in Ghana. PLoS One. 2018;13(10):e0204410.

29. Henshaw SK, Adewole I, Singh S, Bankole A, Oye-Adeniran B, Hussain R. Severity and cost of unsafe abortion complications treated in Nigerian hospitals. Int Fam Plan Perspect. 2008;34(1):40-50.

30. Honda A, Randaoharison PG, Matsui M. Affordability of emergency obstetric and neonatal care at public hospitals in Madagascar. Reprod Health Matters. 2011;19(37):10-20.

31. Ilboudo PG, Greco G, Sundby J, Torsvik G. Costs and consequences of abortions to women and their households: a cross-sectional study in Ouagadougou, Burkina Faso. Health Policy Plan. 2015;30(4):500-7.

32. Ilboudo PG, Greco G, Sundby J, Torsvik G. Estimating the costs for the treatment of abortion complications in two public referral hospitals: a crosssectional study in Ouagadougou, Burkina Faso. BMC Health Serv Res. 2016; 16(1):559.

33. Johns B, Hangoma P, Atuyambe L, Faye S, Tumwine M, Zulu C, Levitt M, Tembo T, Healey J, Li R, et al. The costs and cost-effectiveness of a districtstrengthening strategy to mitigate the 3 delays to quality maternal health care: results from Uganda and Zambia. Glob Health Sci Pract. 2019;7(Suppl 1):S104-S122.

34. Kalu-Umeh NN, Sambo MN, Idris SH, Kurfi AM. Costs and patterns of financing maternal health Care Services in Rural Communities in northern Nigeria: evidence for designing National fee Exemption Policy. Int J MCH AIDS. 2013;2(1):163-72.

35. Kowalewski M, Mujinja $P$, Jahn A. Can mothers afford maternal health care costs? User costs of maternity services in rural Tanzania. Afr J Reprod Health. 2002;6(1):65-73

36. Kruk ME, Mbaruku G, Rockers PC, Galea S. User fee exemptions are not enough: out-of-pocket payments for 'free' delivery services in rural Tanzania. Trop Med Tnt Health. 2008;13(12):1442-51.

37. Le HH, Connolly MP, Yu JB, Pinchevsky Y, Steyn PS: The public health and economic consequences of unintended pregnancies in South Africa. Healthcare in Low-Resource Settings; 2015;3(1). https://doi.org/10.4081/hls. 2015.5258.

38. Levin A, McEuen M, Dmytraczenko T, Ssengooba F, Mangani R, Van Dyck G. Costs of maternal health Care Services in Three Anglophone African Countries. In: Special Initiatives report 22. Bethesda: Partnerships for Health Reform Project, Abt Associates Inc; 2000.

39. Lince-Deroche N, Constant D, Harries J, Blanchard K, Sinanovic E, Grossman D. The costs of accessing abortion in South Africa: Women's costs associated with second-trimester abortion services in Western Cape Province. Contraception. 2015;92(4):339-44.
40. Lince-Deroche N, Constant D, Harries J, Kluge J, Blanchard K, Sinanovic E, Grossman D. The costs and cost effectiveness of providing second-trimester medical and surgical safe abortion services in Western Cape Province, South Africa. PLoS One. 2018;13(6):e0197485.

41. Lince-Deroche N, Fetters T, Sinanovic E, Blanchard K. Accessing medical and surgical first-trimester abortion services: women's experiences and costs from an operations research study in KwaZulu-Natal Province, South Africa. Contraception. 2017;96(2):72-80.

42. Lince-Deroche N, Fetters T, Sinanovic E, Devjee J, Moodley J, Blanchard K. The costs and cost effectiveness of providing first-trimester, medical and surgical safe abortion services in KwaZulu-Natal Province, South Africa. PLoS One. 2017;12(4):e0174615.

43. Lofgren J, Mulowooza J, Nordin P, Wladis A, Forsberg BC. Cost of surgery in a low-income setting in eastern Uganda. Surgery (United States). 2015; 157(6):983-91.

44. Meda IB, Baguiya A, Ridde V, Ouedraogo HG, Dumont A, Kouanda S. Out-ofpocket payments in the context of a free maternal health care policy in Burkina Faso: a national cross-sectional survey. Heal Econ Rev. 2019;9(1):11.

45. Ministry of Health, African Population and Health Research Center, Ipas. The Costs of Treating Unsafe Abortion Complications in Public Health Facilities in Kenya. Nairobi: APHRC; 2018.

46. Moore AM, Dennis M, Anderson R, Bankole A, Abelson A, Greco G, Vwalika B. Comparing women's financial costs of induced abortion at a facility vs. seeking treatment for complications from unsafe abortion in Zambia. Reprod Health Matters. 2018;26(52):1522195.

47. Ntambue AM, Malonga FK, Dramaix-Wilmet M, llunga TM, Musau AN, Matungulu CM, Cowgill KD, Donnen P. Commercialization of obstetric and neonatal care in the Democratic Republic of the Congo: a study of the variability in user fees in Lubumbashi, 2014. PLoS One. 2018;13(10): e0205082.

48. Odhiambo J, Ruhumuriza J, Nkurunziza T, Riviello R, Shrime M, Lin Y, Rusangwa C, Omondi JM, Toma G, Nyirimodoka A, et al. Health facility cost of Cesarean delivery at a Rural District Hospital in Rwanda Using TimeDriven Activity-Based Costing. Matern Child Health J. 2019;23(5):613-22.

49. Orach CG, Dubourg D, De Brouwere V. Costs and coverage of reproductive health interventions in three rural refugee-affected districts, Uganda. Tropical Med Int Health. 2007;12(3):459-69.

50. Parmar D, Leone T, Coast E, Murray SF, Hukin E, Vwalika B. Cost of abortions in Zambia: a comparison of safe abortion and post abortion care. Global Public Health. 2017;12(2):236-49.

51. Paul M, Gebreselassie H, Samai M, Benson J, Kargbo SA, Lazzarino MM Unsafe Abortion in Sierra Leone: An Examination of Costs and Burden of Treatment on Healthcare Resources. J Womens Health Care. 2015; 4(1000228):2167-0420.

52. Pearson L, Gandhi M, Admasu K, Keyes EB. User fees and maternity services in Ethiopia. Int J Gynaecol Obstet. 2011;115(3):310-5.

53. Perkins $M$, Brazier $E$, Themmen E, Bassane B, Diallo D, Mutunga A, Mwakajonga T, Ngobola O. Out-of-pocket costs for facility-based maternity care in three African countries. Health Policy Plan. 2009;24(4):289-300.

54. Ravit M, Philibert A, Tourigny C, Traore M, Coulibaly A, Dumont A, Fournier $P$. The hidden costs of a free caesarean section policy in West Africa (Kayes region, Mali). Matern Child Health J. 2015;19(8):1734-43.

55. Ridde V, Kouanda S, Bado A, Bado N, Haddad S. Reducing the medical cost of deliveries in Burkina Faso is good for everyone, including the poor. PLoS One. 2012;7(3):e33082.

56. Sambo MN, Abdulrazaq GA, Shamang AF, Ibrahim AA. Household cost of antenatal care and delivery services in a rural community of Kaduna state, northwestern Nigeria. Nig Med J. 2013;54(2):87-91.

57. Sicuri E, Bardaji A, Sigauque B, Maixenchs M, Nhacolo A, Nhalungo D, Macete E, Alonso PL, Menendez C. Costs Associated with Low Birth Weight in a Rural Area of Southern Mozambique. PLoS One. 2011;6(12):e28744.

58. Sundaram A, Vlassoff M, Mugisha F, Bankole A, Singh S, Amanya L, Onda T. Documenting the individual- and household-level cost of unsafe abortion in Uganda. Int Perspect Sex Reprod Health. 2013;39(4):174-84.

59. Tongo OO, Orimadegun AE, Ajayi SO, Akinyinka OO. The economic burden of preterm/very low birth weight care in Nigeria. J Trop Pediatr. 2009;55(4): 262-4.

60. Vlassoff M, Fetters T, Kumbi S, Singh S. The health system cost of postabortion care in Ethiopia. Int J Gynecol Obstet. 2012;118(SUPPL. 2): S127-33. 
61. Vlassoff M, Mugisha F, Sundaram A, Bankole A, Singh S, Amanya L, Kiggundu C, Mirembe $F$. The health system cost of post-abortion care in Uganda. Health Policy Plan. 2014;29(1):56-66.

62. Vlassoff M, Musange SF, Kalisa IR, Ngabo F, Sayinzoga F, Singh S, Bankole A. The health system cost of post-abortion care in Rwanda. Health Policy Plan. 2015;30(2):223-33.

63. Witter S, Dieng T, Mbengue D, Moreira I, De Brouwere V. The national free delivery and caesarean policy in Senegal: evaluating process and outcomes. Health Policy Plan. 2010;25(5):384-92.

64. Vlassoff M, Walker D, Shearer J, Newlands D, Singh S. Estimates of health care system costs of unsafe abortion in Africa and Latin America. Int Perspect Sex Reprod Health. 2009;35(3):114-21.

65. Singh S, Darroch JE, Ashford LS. Adding it up: The costs and benefits of investing in sexual and reproductive health 2014. New York: Guttmacher Institute.

66. Dzakpasu S, Powell-Jackson T, Campbell OM. Impact of user fees on maternal health service utilization and related health outcomes: a systematic review. Health Policy Plan. 2014;29(2):137-50.

67. Kyei-Nimakoh M, Carolan-Olah M, McCann TV. Access barriers to obstetric care at health facilities in sub-Saharan Africa-a systematic review. Syst Rev. 2017:6(1):110.

68. Karatasli V, Kanmaz AG, Inan AH, Budak A, Beyan E. Maternal and neonatal outcomes of adolescent pregnancy. J Gynecol Obstet Human Reprod. 2019; 48(5):347-50.

69. Althabe F, Moore IL, Gibbons L, Berrueta M, Goudar SS, Chomba E, Derman RJ, Patel A, Saleem S, Pasha O, et al. Adverse maternal and perinatal outcomes in adolescent pregnancies: The Global Network's Maternal Newborn Health Registry study. Reprod Health. 2015;12(Suppl 2):S8.

70. Neal S, Matthews Z, Frost M, Fogstad H, Camacho AV, Laski L. Childbearing in adolescents aged 12-15 years in low resource countries: a neglected issue. New estimates from demographic and household surveys in 42 countries. Acta Obstet Gynecol Scand. 2012;91(9):1114-8.

\section{Publisher's Note}

Springer Nature remains neutral with regard to jurisdictional claims in published maps and institutional affiliations.

Ready to submit your research? Choose BMC and benefit from:

- fast, convenient online submission

- thorough peer review by experienced researchers in your field

- rapid publication on acceptance

- support for research data, including large and complex data types

- gold Open Access which fosters wider collaboration and increased citations

- maximum visibility for your research: over $100 \mathrm{M}$ website views per year

At $\mathrm{BMC}$, research is always in progress.

Learn more biomedcentral.com/submissions 